\title{
Sensitive limits on the abundance of cold water vapor in the DM Tauri protoplanetary disk ${ }^{\star}$
}

\author{
E. A. Bergin ${ }^{1}$, M. R. Hogerheijde ${ }^{2}$, C. Brinch ${ }^{2}$, J. Fogel ${ }^{1}$, U. A. Yıldız ${ }^{2}$, L. E. Kristensen ${ }^{2}$, E. F. van Dishoeck ${ }^{2,3}$,
} T. A. Bell ${ }^{8}$, G. A. Blake ${ }^{4}$, J. Cernicharo ${ }^{5}$, C. Dominik ${ }^{6,7}$, D. Lis $^{8}$, G. Melnick ${ }^{9}$, D. Neufeld ${ }^{10}$, O. Panić ${ }^{11}$, J. C. Pearson ${ }^{12}$, R. Bachiller ${ }^{13}$, A. Baudry ${ }^{14}$, M. Benedettini ${ }^{15}$, A. O. Benz ${ }^{16}$, P. Bjerkeli ${ }^{17}$, S. Bontemps ${ }^{18}$, J. Braine ${ }^{19}$, S. Bruderer ${ }^{16}$, P. Casellii 19,15 , C. Codella ${ }^{15}$, F. Daniel ${ }^{5}$, A. M. di Giorgio ${ }^{14}$, S. D. Doty ${ }^{20}$, P. Encrenaz ${ }^{21}$, M. Fich ${ }^{22}$, A. Fuente ${ }^{23}$, T. Giannini ${ }^{24}$, J. R. Goicoechea ${ }^{5}$, Th. de Graauw ${ }^{25}$, F. Helmich ${ }^{25}$, G. J. Herczeg ${ }^{3}$, F. Herpin ${ }^{14}$, T. Jacq ${ }^{15}$, D. Johnstone ${ }^{26,27}$, J. K. Jørgensen ${ }^{28}$, B. Larsson ${ }^{29}$, R. Liseau ${ }^{17}$, M. Marseille ${ }^{25}$, C. M Coey $^{22,30}$, B. Nisini ${ }^{14}$, M. Olberg ${ }^{17}$, B. Parise ${ }^{31}$, R. Plume ${ }^{32}$, C. Risacher ${ }^{25}$, J. Santiago-García ${ }^{33}$, P. Saraceno ${ }^{14}$, R. Shipman ${ }^{25}$, M. Tafalla $^{13}$, T. A. van Kempen ${ }^{9}$, R. Visser ${ }^{2}$, S. F. Wampfler ${ }^{16}$, F. Wyrowski ${ }^{31}$, F. van der Tak ${ }^{25,34}$, W. Jellema ${ }^{25}$, A. G. G. M. Tielens ${ }^{2}$, P. Hartogh ${ }^{35}$, J. Stützki ${ }^{36}$, and R. Szczerba ${ }^{37}$

(Affiliations are available on page 5 of the online edition)

Received 31 May 2010 / Accepted 26 June 2010

\section{ABSTRACT}

We performed a sensitive search for the ground-state emission lines of ortho- and para-water vapor in the DM Tau protoplanetary disk using the Herschel/HIFI instrument. No strong lines are detected down to $3 \sigma$ levels in $0.5 \mathrm{~km} \mathrm{~s}^{-1}$ channels of $4.2 \mathrm{mK}$ for the $1_{10}-1_{01}$ line and $12.6 \mathrm{mK}$ for the $1_{11}-0_{00}$ line. We report a very tentative detection, however, of the $1_{10}-1_{01}$ line in the wide band spectrometer, with a strength of $T_{\mathrm{mb}}=2.7 \mathrm{mK}$, a width of $5.6 \mathrm{~km} \mathrm{~s}^{-1}$ and an integrated intensity of $16.0 \mathrm{mK} \mathrm{km} \mathrm{s}^{-1}$. The latter constitutes a $6 \sigma$ detection. Regardless of the reality of this tentative detection, model calculations indicate that our sensitive limits on the line strengths preclude efficient desorption of water in the UV illuminated regions of the disk. We hypothesize that more than 95-99\% of the water ice is locked up in coagulated grains that have settled to the midplane.

Key words. ISM: abundances - ISM: molecules - protoplanetary disks

\section{Introduction}

Because of its association with biology on Earth, water is one of the most important molecules in the solar system and beyond. However, the origin of water on Earth is highly uncertain. What is clear is that the distribution of hydrated rocks in the solar system (Abe et al. 2000) suggests that water resided in the vapor phase in the warm $\left(T_{\text {dust }}>100 \mathrm{~K}\right)$ inner solar nebula and is predominantly condensed in the form of ice beyond the so-called snow-line between 2-2.5 AU (Hayashi 1981; Abe et al. 2000).

The observation of cool water vapor in protoplanetary disks is hampered by atmospheric attenuation and requires spacebased observations. A number of recent detections of hot $\left(T_{\text {gas }}>\right.$ $200 \mathrm{~K}$ ) water by the Spitzer Space Telescope has demonstrated that abundant water vapor is a ubiquitous component in protoplanetary disks (Salyk et al. 2008; Carr \& Najita 2008; Pontoppidan et al. 2010). However, this emission likely arises within the snow-line of these young systems and therefore does not provide a complete picture of the distribution of water (both ice and vapor) in disks with sizes in excess of 100 AU. In particular, these observations do not probe the cold $\left(T_{\text {dust }} \sim T_{\text {gas }} \sim\right.$ $20 \mathrm{~K}$ ) outer parts of the disk where most of the mass resides.

* Herschel is an ESA space observatory with science instruments provided by European-led Principal Investigator consortia and with participation important from NASA.
The Herschel Space Observatory (Pilbratt et al. 2010) offers a new opportunity for a characterization of the distribution and evolution of water vapor in protoplanetary disks. We report here a sensitive search for cold water emission in the ground-state lines of ortho- $(o)$ and para- $(p) \mathrm{H}_{2} \mathrm{O}$ using the high spectral resolution of the HIFI instrument (de Graauw et al. 2010) towards the well studied DM Tau protoplanetary disk. This study is part of the guaranteed time key program "Water In Star forming regions" (van Dishoeck et al. in prep.). Stringent limits to the strength of both lines of a few $\mathrm{mK}$ suggest that the outer regions of the disk contain little water vapor or water ice. In Sect. 2 we outline the observations. Section 3 presents results from detailed modeling; Sect. 4 summarizes the implications of our result.

\section{Observations and results}

DM Tau is a T Tauri star located at $\alpha(2000)=4^{\mathrm{h}} 33^{\mathrm{m}} 48^{\mathrm{s}} .7$ and $\delta(2000)=18^{\circ} 10^{\prime} 10^{\prime \prime}$ with a disk diameter, estimated from $\mathrm{CO}$ emission, of $\sim 1800 \mathrm{AU}$ at a systemic velocity of $6 \mathrm{~km} \mathrm{~s}^{-1}$ and $i \sim-35^{\circ}$ (Piétu et al. 2007). The source is a single M1 star (White \& Ghez 2001) with $L=0.25 L_{\odot}$. DM Tau has a chemically rich molecular disk (Dutrey et al. 1997). Accretion from the disk to the star also provides a source of excess UV luminosity, with an overall UV field strength of $G_{0} \sim 240$ (relative to the standard interstellar radiation field, ISRF; Bergin et al. 2003, 2004; Habing 1968). The object is a transition disk with an inner 
hole on the order of a few AU, based on models of Spitzer spectra (Calvet et al. 2005).

DM Tau was observed with the HIFI instrument using the double beam switch observing mode with a throw of 3'.0. On 2010 March 22 spectra were taken in receiver band $1 \mathrm{~b}$ with an on-source integration time of $198 \mathrm{~min}$, and $T_{\text {sys }}=78-96 \mathrm{~K}$. On 2010 March 4 spectra were taken in receiver band $4 \mathrm{~b}$ and an on-source integration time of $328 \mathrm{~min}$, and $T_{\text {sys }}=370-410 \mathrm{~K}$.

The HIFI beam of $39^{\prime \prime}$ at $556 \mathrm{GHz}$ and $21^{\prime \prime}$ at $1113 \mathrm{GHz}$ is larger than the DM Tau disk with a diameter of $12^{\prime \prime} .7$ at $140 \mathrm{pc}$. The beams are also larger than the pointing accuracy of Herschel of $\sim 2^{\prime \prime}$. The data were recorded with wide band spectrometer (WBS) covering 4.4 GHz with $1.1 \mathrm{MHz}$ resolution $\left(0.59\right.$ and $0.30 \mathrm{~km} \mathrm{~s}^{-1}$ at 556 and $1113 \mathrm{GHz}$, respectively), and the high-resolution spectrometer (HRS) covering $230 \mathrm{MHz}$ at $0.25 \mathrm{MHz}$ resolution $\left(0.13\right.$ and $0.067 \mathrm{~km} \mathrm{~s}^{-1}$ at 556 and $1113 \mathrm{GHz}$, respectively). Both $\mathrm{H}$ - and V-polarizations were measured.

The raw data were calibrated onto the $T_{\mathrm{A}}^{*}$ scale by the inorbit system and converted to $T_{\mathrm{mb}}$ assuming a beam efficiency of 0.74 The data were reduced using HIPE v3.0. Subsequently, the data were exported to CLASS ${ }^{1}$. The HIFI flux calibration is accurate to $10 \%$, while the velocity scale of HIPE v3.0 is accurate to several $\mathrm{m} \mathrm{s}^{-1}$. For both lines, the WBS data were rebinned to $0.54 \mathrm{~km} \mathrm{~s}^{-1}$ channels (or $1.0 \mathrm{MHz}$, close to the instrumental resolution of $1.1 \mathrm{MHz}$ ); the HRS data were rebinned to $0.45 \mathrm{~km} \mathrm{~s}^{-1}$ channels. All spectra, including the the $\mathrm{H}$ - and V-polarizations, were averaged together weighted by their respective noise levels. The resulting rms noise levels are $2.9 \mathrm{mK}$ (HRS) and $1.4 \mathrm{mK}$ (WBS) for the $\mathrm{H}_{2} \mathrm{O}_{101}-1_{01}$ line, and $7.2 \mathrm{mK}$ (HRS) and $4.3 \mathrm{mK}$ (WBS) for the $\mathrm{H}_{2} \mathrm{O} 1_{11}-0_{00}$ line $^{2}$. Figure 1 illustrates for band $1 \mathrm{~b}$ that the noise in our data decreases as (time) $)^{-0.5}$ up to the full achieved integration times.

Figure 2 presents the HRS and WBS spectra of the two water transitions. No strong lines are detected; for comparison, Fig. 2 also shows the ${ }^{12} \mathrm{CO} 1-0$ spectrum of DM Tau (Kessler-Silacci 2004; Panić et al., in prep.) showing a clear emission line with a width of $\sim 2 \mathrm{~km} \mathrm{~s}^{-1}$ centered on the source velocity of $+6.1 \mathrm{~km} \mathrm{~s}^{-1}$. In the WBS spectrum of the $\mathrm{H}_{2} \mathrm{O} 1_{10}-1_{01}$ line a weak feature is present between $V_{\mathrm{LSR}}+0.5$ and $+10 \mathrm{~km} \mathrm{~s}^{-1}$, peaking around $+6.6 \mathrm{~km} \mathrm{~s}^{-1}$; a similar feature is seen in the noisier HRS spectrum. With $T_{\mathrm{mb}}=4.3 \mathrm{mK}$, the brightest channel lies at $3 \sigma$. Integrated between +0.5 and $+10 \mathrm{~km} \mathrm{~s}^{-1}$, the feature contains $17.2 \pm 3.2 \mathrm{mK} \mathrm{km} \mathrm{s}^{-1}$, a $5 \sigma$ result. A Gaussian fit to the feature yields best fit parameters of $V_{\mathrm{LSR}}=+6.8 \pm 0.7 \mathrm{~km} \mathrm{~s}^{-1}$, a $F W H M$ width of $5.6 \pm 1.2 \mathrm{~km} \mathrm{~s}^{-1}$, an intensity $T_{\mathrm{mb}}=2.7 \pm 1.4 \mathrm{mK}$, and an integrated intensity of $16.0 \pm 2.7 \mathrm{mK} \mathrm{km} \mathrm{s}^{-1}(6 \sigma)$.

Arguments in favor of interpreting this feature as a positive detection of the $\mathrm{H}_{2} \mathrm{O} 1_{10}-1_{01}$ line include the facts that the integrated intensity constitutes a 5-6 $\sigma$ detection and that the feature peaks near the systemic velocity of $6.1 \mathrm{~km} \mathrm{~s}^{-1}$. Against the interpretation as a positive detection is a line peak that is only $2-3 \sigma$ and a linewidth which is twice that of the ${ }^{12} \mathrm{CO}$ line. The width would suggest that the emission arises from within $10 \mathrm{AU}$. With respect to the latter, it is interesting that $\mathrm{HCO}^{+} 1-0$ line has a blue wing extending over $\sim 3 \mathrm{~km} \mathrm{~s}^{-1}$ (Dutrey et al. 1997).

\footnotetext{
${ }^{1}$ http://wWw.iram.fr/IRAMFR/GILDAS

2 Although the channel spacing in both bands in similar (0.54 vs. $0.45 \mathrm{~km} \mathrm{~s}^{-1}$ ), the noise in the WBS is 1.7-2.0 times lower than the noise in the HRS because of the larger noise bandwidth of the WBS and a $\sqrt{2}$ loss factor in the HRS autocorrelator.
}

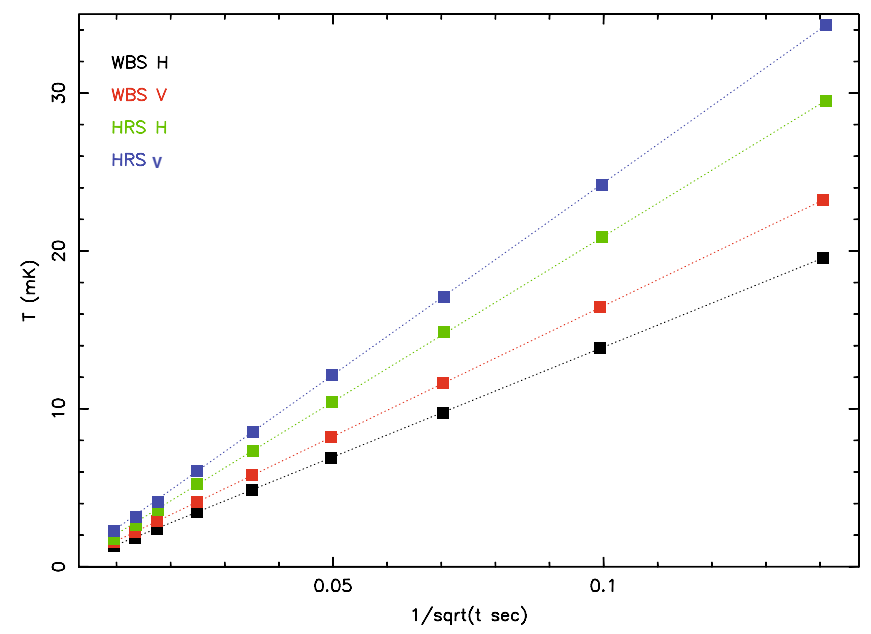

Fig. 1. Noise level (rms) vs integration time of the WBS and HRS data of the $\mathrm{H}_{2} \mathrm{O} 1_{10}-1_{01}$ line. The noise decreases as $\sqrt{t_{\text {int }}}$.

Neither set of arguments is clearly stronger, and we interpret the feature in the $\mathrm{H}_{2} \mathrm{O} 1_{10}-1_{01}$ spectrum as a very tentative detection of water vapor in the disk of DM Tau. In the remainder of this Letter, we will work with an intensity of $\$ 2.7 \mathrm{mK}$ for the $\mathrm{H}_{2} \mathrm{O} 1_{10}-1_{01}$ line and with an upper limit to the intensity of the $\mathrm{H}_{2} \mathrm{O} 1_{11}-0_{00}$ line of $<12.6 \mathrm{mK}$.

Smoothing the WBS spectra to $\sim 27 \mathrm{~km} \mathrm{~s}^{-1}$ resolution results in positive detections of the continuum of DM Tau at $556.9 \mathrm{GHz}$ of $3.0 \pm 0.5 \mathrm{mK}(0.6 \pm 0.1 \mathrm{Jy})$ and at $1113.3 \mathrm{GHz}$ of $5.7 \pm 0.9 \mathrm{mK}$ $(1.3 \pm 0.2 \mathrm{Jy})$. These values are consistent with other continuum measurements (Dutrey et al. 1996).

\section{Model predictions}

\subsection{Chemistry of water vapor in the cold outer disk}

Because the midplane temperatures are well below the evaporation temperature at densities representative of the midplane of $>150 \mathrm{~K}$ (Fraser et al. 2001; D’Alessio et al. 2005), we can expect that beyond the snow-line water is mostly frozen on the surfaces of dust grains. Therefore, molecular emission arises predominantly from the warm disk surface that is heated by stellar irradiation (Aikawa et al. 2002). Beyond $10 \mathrm{AU}$ the dust in this superheated layer is heated to $<100 \mathrm{~K}$ (Nomura et al. 2007) and again it is anticipated that water will remain as ice. However, the upper layers of the disk are exposed to energetic X-ray and FUV radiation which provide a source for nonthermal desorption. Models of X-ray induced desorption suggest that X-rays cannot release significant $\mathrm{H}_{2} \mathrm{O}$ into the gas (Najita et al. 2001). A more profitable method to desorb water ice in the cold outer disk is via photodesorption, which has a measured yield (molecules/photon) of $\sim 10^{-3}$ (Öberg et al. 2009). This has been suggested as providing a basal column of water vapor in the disk by Dominik et al. (2005).

Figure 3 presents results from a detailed calculation of the UV radiation transfer and chemistry of a standard T Tauri disk with $M_{\text {disk }}=0.03 M_{\odot}, R_{\text {out }}=400$ AU and a standard gas-todust ratio (Fogel et al. 2010). A key aspect in the calculation of the importance of photodesorption is the formation of water ice on the grain surface via oxygen hydrogenation and also the 2D propagation of UV photons. This includes Ly $\alpha$ radiation. which is important for $\mathrm{H}_{2} \mathrm{O}$ (van Dishoeck et al. 2006). As can be seen a thin layer exists where water vapor is present in moderate abundance $\left(\sim 10^{-7}-10^{-6}\right)$ in the cold outer regions of the disk.

As noted by Dominik et al. (2005) the column density of water vapor produced by photodesorption is independent of the 


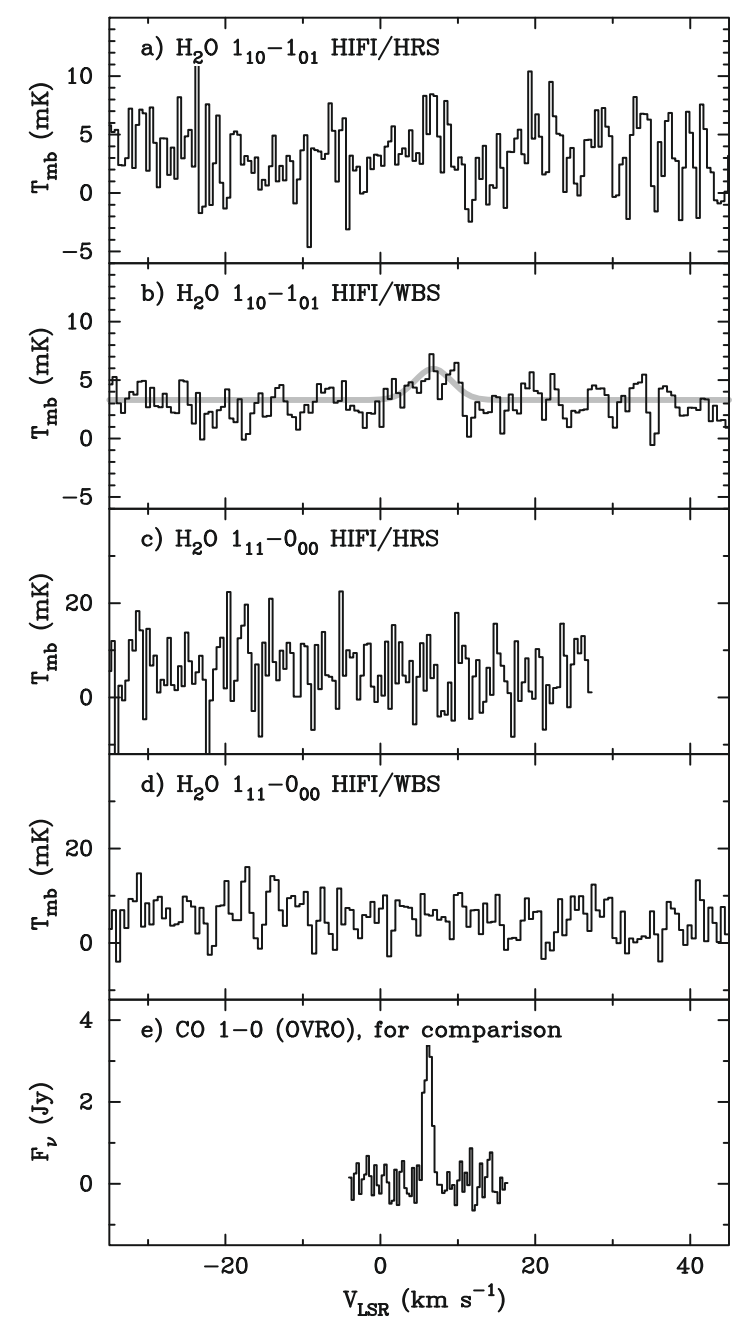

Fig. 2. a)-d) $\mathrm{H}_{2} \mathrm{O}$ spectra obtained with HIFI. A tentative feature is present in panel b) for $\mathrm{H}_{2} \mathrm{O} 1_{10}-1_{01}$ detected with WBS between $V_{\text {LSR }}$ of +0.5 and $+10 \mathrm{~km} \mathrm{~s}^{-1}$. A Gaussian fit to the feature is shown by the smooth grey line. The HRS data on this line in panel (a) are consistent with this result but noisier. Only noise is seen in panels c) and d) showing the $1_{11}-0_{00}$ line. e) Comparison ${ }^{12} \mathrm{CO} 1-0$ spectrum of DM Tau obtained with OVRO (Kessler-Silacci 2004; Panić et al., in prep.).

photon flux. This can be seen by balancing formation by photodesorption with destruction by UV photodissociation, giving a maximum water abundance (Hollenbach et al. 2009): $n\left(\mathrm{H}_{2} \mathrm{O}\right) \int \sigma_{\lambda} F_{\lambda} \mathrm{d} \lambda=F_{\mathrm{UV}} Y \sigma_{\text {site }} N_{\mathrm{p}} f_{\text {ice }} N_{\mathrm{m}}^{-1} n\left(\mathrm{H}_{2} \mathrm{O}\right)_{\text {ice }}$. Here $F_{\mathrm{UV}}=$ $\int F_{\lambda} \mathrm{d} \lambda$ is the integral of the photon flux given in Fig. 3 and $\sigma_{\lambda} \sim \sigma_{\text {Ly } \alpha} \sim 10^{-17} \mathrm{~cm}^{-2}$ (van Dishoeck et al. 2006). $\sigma_{\text {site }}$ is the cross section of a given site on the grain $\left(=\pi a^{2} / N_{\text {site }}\right.$, with grain radius $a=0.1 \mu \mathrm{m}$ and $\left.N_{\text {site }}=10^{6}\right) . N_{\mathrm{p}}=2$ is a correction for the fact the UV photons only penetrate the first few monolayers (Öberg et al. 2009), with a yield of $Y \sim 2 \times 10^{-3} \cdot f_{\text {ice }}$ is the fraction of water ice over the total amount of ice and $N_{\mathrm{m}}$ is the number of monolayers. Based on this approximation we find $x\left(\mathrm{H}_{2} \mathrm{O}\right)_{\max } \sim 10^{-6}$; for scaling relations see Hollenbach et al. (2009). This could be lowered if the grains have a reduced fraction of water ice or perhaps less than a monolayer of coverage.

\subsection{Comparison to observations}

Using the above chemical model calculation as input, we use the molecular excitation and radiative transfer code LIME (Brinch et al., in prep.) to calculate the line intensity in both observed

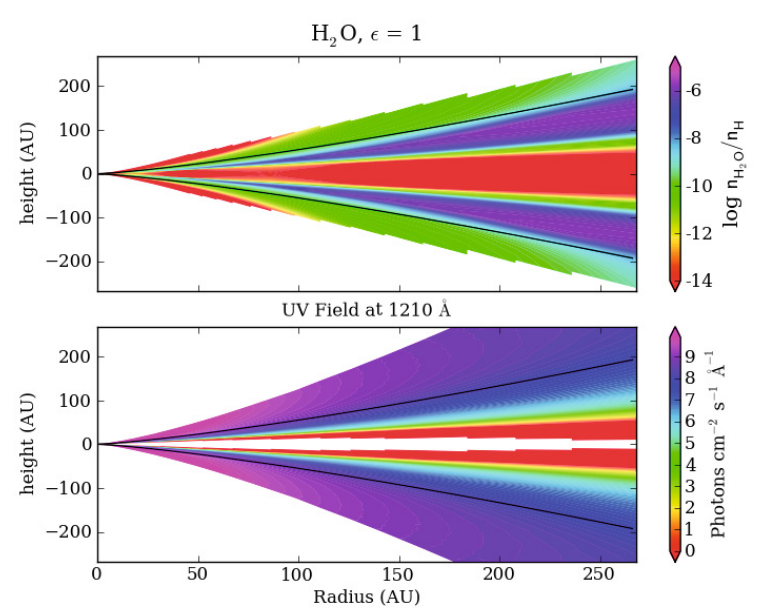

Fig. 3. Abundance of water vapor (Top) and Ly $\alpha$ photon flux (Bottom) shown as a function of both radial distance and vertical height. The water vapor abundance is relative to total hydrogen and is only plotted for points where the vertical height to radial distance ratio is less than 1. The line in both plots refers to the $\tau=1$ surface for stellar radiation and $\epsilon=1$ refers to the fact that these models were run with a standard dust to gas ratio. All model results taken from Fogel et al. (2010).

water lines. We use the collision rates of water with $p-\mathrm{H}_{2}$ from Faure et al. (2007) as provided by the LAMDA database (Schöier et al. 2005) ${ }^{3}$ and convolved the results with the appropriate Herschel beams. In our models we assume an intrinsic broadening of $0.4 \mathrm{~km} \mathrm{~s}^{-1}$ on top of a Keplerian velocity profile.

Because of the appreciable abundance of $\mathrm{H}_{2} \mathrm{O}$ in the model (disk-averaged column density of $6 \times 10^{14} \mathrm{~cm}^{-2}$ ), it is not surprising that significant line intensities are predicted of $T_{\mathrm{mb}}=$ $140 \mathrm{mK}$ for the $1_{10}-1_{01}$ line and $300 \mathrm{mK}$ for the $1_{11}-0_{00}$ line with a Gaussian spectral profile. Clearly, our observations rule out the presence of the amounts of water vapor predicted by photodesorption regardless of the details of our model.

Absorption by low-excitation water from foreground material cannot explain the absence of detected emission: the cloud seen in ${ }^{12} \mathrm{CO}$ by (Dutrey et al. 1997) has narrow emission centered $3 \mathrm{~km} \mathrm{~s}^{-1}$ away from the DM Tau disk. Only when the column density of water is scaled down by a factor of 130 to a disk-averaged value of $5 \times 10^{12} \mathrm{~cm}^{-2}$ does the predicted strength of the $1_{10}-1_{01}$ line becomes consistent with the observed limits; the limits on the $1_{11}-0_{00}$ line are less strict because of the higher noise of these observations.

Our model could predict lines that are too intense if we overestimate the collisional excitation of water. Dick et al. (2010) suggested that existing collisional excitation rates for water are overestimated at temperatures below $\sim 50-80 \mathrm{~K}$. Decreasing the collisional excitation rates has little effect on the line strengths for the original column density. In that case, the lines are still highly optically thick, with maximum optical depth of 2000, and line trapping effectively excites the line. However, for collision rates lower by a factor of 10 compared to the adopted rates, reducing the disk averaged column density by a factor of 20 to $3 \times 10^{13} \mathrm{~cm}^{-2}$ is sufficient to comply with the observational constraints.

Our generic model disk contains $0.03 M_{\odot}$ similar to DM Tau, but its $400 \mathrm{AU}$ radius is only half that of DM Tau. The increased beam dilution only strengthens our conclusions. For a DM Tau specific model, Dominik et al. (2005) predict the $1_{10}-1_{01}$ line

3 http://www.strw.leidenuniv.nl/ moldata 
to be in absorption. Line profiles with combinations of emission and strong absorption naturally arise in disk models with strong temperature, density and water abundance gradients viewed under non-zero inclinations. An example is provided by Cernicharo et al. (2009) for the HD 97048 disk. These models have water abundances peaking at much larger height above the midplane, explaining the low excitation resulting in strong absorption. For our discussion here, we stress that all these models predict emission or absorption lines that are inconsistent with our observational limits. Future work will focus on more detailed models for DM Tau, and also explore the effect of dust settling and nonstandard gas-to-dust ratios (Brinch et al. in prep.).

Finally, we note that Ceccarelli et al. (2005) have claimed a $4 \sigma$ detection HDO in the DM Tau. This detection has been cast into doubt based on line formation considerations (Guilloteau et al. 2006). Our models predict water will be in emission, and not absorption. At face value this would argue against the reality of the absorption. If we derive a column density from the HDO observations and the limits here, the $\mathrm{D} / \mathrm{H}$ ratio would also be exceedingly high $>0.2$. Deeper HDO observations are needed to settle this issue.

\section{Implications}

We have presented the results from a deep search for the ground state emission lines of $o-\mathrm{H}_{2} \mathrm{O}$ and $p-\mathrm{H}_{2} \mathrm{O}$ towards the DM Tau disk. Based on the best theoretical knowledge we have to date, water vapor should be present in the outer disk and presumably emissive. However, our sensitive observations show that, at least for this object, it is not. Our limit on the $p-\mathrm{H}_{2} \mathrm{O}$ line precludes an extreme $o / p$ ratio as explanation for the low $o-\mathrm{H}_{2} \mathrm{O}$ emission strength. There are two potential explanations for this result. Either water vapor is unemissive as would be the case if the excitation at low temperature is lower than generally assumed (Dick et al. 2010). Cernicharo et al. (2009) also show that the water excitation sensitively depends on the adopted collision rates with $o$ - and $p-\mathrm{H}_{2}$. However, our calculations suggest that line trapping is sufficiently effective at the predicted water abundances to still produce detectable lines. Alternatively, our physi$\mathrm{cal} / \mathrm{chemical}$ understanding may be incorrect. The photodesorption yield is measured in the laboratory at low temperature and the abundance is independent of the photon flux so these aspects appear unlikely to provide the answer. One intriguing possibility is that the upper layers of the outer disk are "dry" which could be the case if only bare grains are present in the region where UV photons are present. A well known key aspect of disk physical evolution is the coagulation and settling of dust grains to the disk midplane (Weidenschilling \& Cuzzi 1993; Dullemond \& Dominik 2004; Furlan et al. 2006). Icy grains present a more favorable surface for grain coagulation and would therefore become larger and settle to deeper layers than their bare silicate counterparts (Dominik \& Tielens 1997). While upward mixing of gas and small grains may occur, larger ice-bearing grains remain in the midplane. In addition, the total $n_{\text {grain }} \sigma_{\text {grain }}$ will be reduced, thereby reducing the efficiency of grain surface formation of $\mathrm{H}_{2} \mathrm{O}$ upon which the photodesorption model depends for water vapor creation (Hollenbach et al. 2009). This "cold-finger" effect was also proposed by Meijerink et al. (2009) to explain the truncation of warm water vapor beyond $\sim 1$ AU seen in Spitzer measurements. Thus, Herschel and Spitzer both suggest that the disk around DM Tau is settled. In summary, our Herschel results suggest that less than $1-5 \%$ of the water ice reservoir survives in the UV-illuminated outer disk regions around DM Tau. If this finding is confirmed by more detailed models and by additional observations, Herschel may be telling us something entirely new about the chemical structure of protoplanetary disks.

Acknowledgements. HIFI has been designed and built by a consortium of institutes and university departments from across Europe, Canada and the United States under the leadership of SRON Netherlands Institute for Space Research, Groningen, The Netherlands and with major contributions from Germany, France and the US. Consortium members are: Canada: CSA, U.Waterloo; France: CESR, LAB, LERMA, IRAM; Germany: KOSMA, MPIfR, MPS; Ireland, NUI Maynooth; Italy: ASI, IFSI-INAF, Osservatorio Astrofisico di Arcetri- INAF; Netherlands: SRON, TUD; Poland: CAMK, CBK; Spain: Observatorio Astronómico Nacional (IGN), Centro de Astrobiología (CSIC-INTA). Sweden: Chalmers University of Technology - MC2, RSS \& GARD; Onsala Space Observatory; Swedish National Space Board, Stockholm University - Stockholm Observatory; Switzerland: ETH Zurich, FHNW; USA: Caltech, JPL, NHSC. Support for this work was provided by NASA through an award issued by JPL/Caltech. E.A.B. acknowledges support by NSF Grant 0707777, M.R.H. by NWO grant 639.042.404.

\section{References}

Abe, Y., Ohtani, E., Okuchi, T., Righter, K., \& Drake, M. 2000, Water in the Early Earth, Origin of the earth and moon, ed. R. M. Canup, K. Righter, et al. (Tucson: University of Arizona Press), 413

Aikawa, Y., van Zadelhoff, G. J., van Dishoeck, E. F., \& Herbst, E. 2002, A\&A, 386, 622

Bergin, E., Calvet, N., D’Alessio, P., \& Herczeg, G. J. 2003, ApJ, 591, L159

Bergin, E., Calvet, N., Sitko, M. L., et al. 2004, ApJ, 614, L133

Calvet, N., D’Alessio, P., Watson, D. M., et al. 2005, ApJ, 630, L185

Carr, J. S. \& Najita, J. R. 2008, Science, 319, 1504

Ceccarelli, C., Dominik, C., Caux, E., Lefloch, B., \& Caselli, P. 2005, ApJ, 631, L81

Cernicharo, J., Ceccarelli, C., Ménard, F., Pinte, C., \& Fuente, A. 2009, ApJ, 703, L123

D'Alessio, P., Calvet, N., \& Woolum, D. S. 2005, in Chondrites and the Protoplanetary Disk, ed. A. N. Krot, E. R. D. Scott, \& B. Reipurth, ASP Conf. Ser., 341, 353

de Graauw, Th., Helmich, F. P., Phillips, T., et al. 2010, A\&A, 518, L6

Dick, M. J., Drouin, B. J., \& Pearson, J. C. 2010, Phys. Rev. A, 81, 022706

Dominik, C., \& Tielens, A. G. G. M. 1997, ApJ, 480, 647

Dominik, C., Ceccarelli, C., Hollenbach, D., \& Kaufman, M. 2005, ApJ, 635, L85

Dullemond, C. P., \& Dominik, C. 2004, A\&A, 421, 1075

Dutrey, A., Guilloteau, S., Duvert, G., et al. 1996, A\&A, 309, 493

Dutrey, A., Guilloteau, S., \& Guelin, M. 1997, A\&A, 317, L55

Faure, A., Crimier, N., Ceccarelli, C., et al. 2007, A\&A, 472, 1029

Fogel, J. K. J., Bethell, T. J., Bergin, E. A., Calvet, N., \& Semenov, D. 2010, ApJ, submitted

Fraser, H. J., Collings, M. P., McCoustra, M. R. S., \& Williams, D. A. 2001, MNRAS, 327, 1165

Furlan, E., Hartmann, L., Calvet, N., et al. 2006, ApJS, 165, 568

Guilloteau, S., Piétu, V., Dutrey, A., \& Guélin, M. 2006, A\&A, 448, L5

Habing, H. J. 1968, Bull. Astron. Inst. Netherlands, 19, 421

Hayashi, C. 1981, Progr. Theor. Phys. Suppl., 70, 35

Hollenbach, D., Kaufman, M. J., Bergin, E. A., \& Melnick, G. J. 2009, ApJ, 690, 1497

Kessler-Silacci, J. 2004, Ph.D. Thesis, Caltech

Meijerink, R., Pontoppidan, K. M., Blake, G. A., Poelman, D. R., \& Dullemond, C. P. 2009 , ApJ, 704, 1471

Najita, J., Bergin, E. A., \& Ullom, J. N. 2001, ApJ, 561, 880

Nomura, H., Aikawa, Y., Tsujimoto, M., Nakagawa, Y., \& Millar, T. J. 2007, ApJ, 661, 334

Öberg, K. I., Linnartz, H., Visser, R., \& van Dishoeck, E. F. 2009, ApJ, 693, 1209

Piétu, V., Dutrey, A., \& Guilloteau, S. 2007, A\&A, 467, 163

Pilbratt, G., Riedinger, J. R., Passvogel, T., et al. 2010, A\&A, 518, L1

Pontoppidan, K. M., Salyk, C., Blake, G. A., et al. 2010, ApJ, accepted [arXiv: 1006.4189]

Salyk, C., Pontoppidan, K. M., Blake, G. A., et al. 2008, ApJ, 676, L49

Schöier, F. L., van der Tak, F. F. S., van Dishoeck, E. F., \& Black, J. H. 2005, A\&A, 432, 369

van Dishoeck, E. F., Jonkheid, B., \& van Hemert, M. C. 2006, in Faraday Discuss., 133, 231

Weidenschilling, S. J., \& Cuzzi, J. N. 1993, in Protostars and Planets III, ed. E. H. Levy, \& J. I. Lunine, 1031

White, R. J., \& Ghez, A. M. 2001, ApJ, 556, 265 
1 Department of Astronomy, The University of Michigan, 500 Church Street, Ann Arbor, MI 48109-1042, USA

e-mail: ebergin@umich.edu

2 Leiden Observatory, Leiden University, PO Box 9513, 2300 RA Leiden, The Netherlands

3 Max Planck Institut für Extraterrestrische Physik, Giessenbachstrasse 1, 85748 Garching, Germany

${ }^{4}$ California Institute of Technology, Division of Geological and Planetary Sciences, MS 150-21, Pasadena, CA 91125, USA

5 Centro de Astrobiología. Departamento de Astrofísica, CSIC-INTA, Carretera de Ajalvir, Km 4, Torrejón de Ardoz, 28850 Madrid, Spain

6 Astronomical Institute Anton Pannekoek, University of Amsterdam, Kruislaan 403, 1098 SJ Amsterdam, The Netherlands

7 Department of Astrophysics/IMAPP, Radboud University Nijmegen, PO Box 9010, 6500 GL Nijmegen, The Netherlands

8 California Institute of Technology, Cahill Center for Astronomy and Astrophysics, MS 301-17, Pasadena, CA 91125, USA

9 Harvard-Smithsonian Center for Astrophysics, 60 Garden Street, MS 42, Cambridge, MA 02138, USA

10 Department of Physics and Astronomy, Johns Hopkins University, 3400 North Charles Street, Baltimore, MD 21218, USA

11 European Southern Observatory, Karl-Schwarzschild-Str. 2, 85748 Garching, Germany

12 Jet Propulsion Laboratory, California Institute of Technology, Pasadena, CA 91109, USA

13 Observatorio Astronómico Nacional (IGN), Calle Alfonso XII 3, 28014 Madrid, Spain

14 INAF - Istituto di Fisica dello Spazio Interplanetario, Area di Ricerca di Tor Vergata, via Fosso del Cavaliere 100, 00133 Roma, Italy

15 INAF - Osservatorio Astrofisico di Arcetri, Largo E. Fermi 5, 50125 Firenze, Italy

16 Institute of Astronomy, ETH Zurich, 8093 Zurich, Switzerland

17 Department of Radio and Space Science, Chalmers University of Technology, Onsala Space Observatory, 43992 Onsala, Sweden

18 Université de Bordeaux, Laboratoire d'Astrophysique de Bordeaux, France; CNRS/INSU, UMR 5804, Floirac, France

19 School of Physics and Astronomy, University of Leeds, Leeds LS2 9JT, UK
20 Department of Physics and Astronomy, Denison University, Granville, OH, 43023, USA

21 LERMA and UMR 8112 du CNRS, Observatoire de Paris, 61 Av. de l'Observatoire, 75014 Paris, France

22 University of Waterloo, Department of Physics and Astronomy, Waterloo, Ontario, Canada

23 Observatorio Astronómico Nacional, Apartado 112, 28803 Alcalá de Henares, Spain

${ }^{24}$ INAF - Osservatorio Astronomico di Roma, 00040 Monte Porzio catone, Italy

25 SRON Netherlands Institute for Space Research, PO Box 800, 9700 AV, Groningen, The Netherlands

26 National Research Council Canada, Herzberg Institute of Astrophysics, 5071 West Saanich Road, Victoria, BC V9E 2E7, Canada

27 Department of Physics and Astronomy, University of Victoria, Victoria, BC V8P 1A1, Canada

${ }^{28}$ Centre for Star and Planet Formation, Natural History Museum of Denmark, University of Copenhagen, Øster Voldgade 5-7, 1350 Copenhagen K., Denmark

29 Department of Astronomy, Stockholm University, AlbaNova, 106 91 Stockholm, Sweden

30 The University of Western Ontario, Department of Physics and Astronomy, London, Ontario, N6A 3K7, Canada

31 Max-Planck-Institut für Radioastronomie, Auf dem Hügel 69, 53121 Bonn, Germany

32 Department of Physics and Astronomy, University of Calgary, Calgary, T2N 1N4, AB, Canada

33 Instituto de Radioastronomía Milimétrica (IRAM), Avenida Divina Pastora 7, Núcleo Central, 18012 Granada, Spain

34 Kapteyn Astronomical Institute, University of Groningen, PO Box 800, 9700 AV, Groningen, The Netherlands

35 Max-Planck-Insitut für Sonnensystemforschung, 37191 KatlenburgLindau, Germany

36 KOSMA, I. Physik. Institut, Universität zu Köln, Zülpicher Str. 77, D 50937 Köln, Germany

37 N. Copernicus Astronomical Center, Rabianska 8, 87-100, Torun, Poland 